\title{
Smart, simulation-based resource sharing in federated production networks
}

\author{
Botond Kádára (2), Péter Egria, Gianfranco Pedonea, Takafumi Chidab,c \\ ${ }^{a}$ Centre of Excellence in Production Informatics and Control, Institute for Computer Science and Control, Hungarian Academy of Sciences, Budapest, Hungary \\ ${ }^{b}$ Hitachi, Ltd. Research \& Development Group \\ ${ }^{c}$ Kobe University, Graduate School of System Informatics, Kobe, Japan
}

The rise of Industry 4.0 and the convergence of the digital and physical worlds increasingly, where not radically, transforms the production networks. The paper presents the preliminary results of a distributed collaboration framework developed with the aim to facilitate the cooperation of various production sites. The objective is to manage a network of manufacturers who can dynamically re-configure and share their resources within a pre-registered community and analyze how the cardinality of the federation influences both the cooperation and the global and local production KPI-s. The novelty of the concept is the combination of the agent-based control, the matching algorithm combined with the detailed digital simulation of the distributed production systems and the collaborative IT platform.

Agent-based manufacturing, Simulation, Network coordination

\section{Introduction}

Even before the rise of the Industry 4.0 paradigm, where the newest Information and Communication Technologies (ICT) are strongly emphasized and deployed into different industrial solutions, the needs for reacting quickly to market changes, being responsive by using decentralized, cooperative structures had already been identified by numerous researchers. In [1], [2] and [3] authors give a large and detailed literature overview about many research aspects of distributed production networks from the past decades.

The concept of a Collaborative Network (CN) has already been developed and described in [4], whose main characteristics are autonomy, geographic distribution and heterogeneity. With the advent of Industry 4.0 major efforts are oriented to integrating together Cyber- Physical Systems, Internet of Things and Cloud Computing in a so-called Smart Factory (SF), where the need for interoperability is higher, as the continuous collaboration of devices, services and humans (through intelligent interfaces) at all levels is extremely significant [5]. The trends and the issues for enterprise integration and interoperability in manufacturing systems are presented in detail in [6].

In [7] the authors investigate collaboration in depth, observing that technology in itself is not enough, organizational innovations are also necessary for increasing productivity. Among other issues, information sharing and resource pooling are emphasized as levers for realising collaboration. Dynamics like work in process (WIP) fluctuations and lead-time values within production networks comprising autonomous work systems with local capacity control are analyzed in [8]. Research about new business models such as sharing concepts for the joint use of resources by different companies are presented and examined in [9]. Here, the evaluations about how different resource sharing mechanisms impact the production network were carried out by a simulation model and a control-theoretic model.
With the idea of Cloud Manufacturing, developed on the basis of Cloud Computing [10], manufacturing resources (assets) can be (to some extent) accessed as cloud services at different levels, from the physical up to enterprise collaboration level. In [11] interoperability was identified as "a key enabler for cloud manufacturing" and proposed a framework called "C-MARS" for realizing interoperability across heterogeneous computer-aided manufacturing systems. Using this framework, manufacturing resources might be shared by a large number of clients based on requirements and priorities but without a common space dedicated to market driven interactions and support in capacity sharing.

In Crowdsourced Manufacturing, considered as a new type of cooperation in production networks, companies or producer entities cooperate within a brokering federation in order to reach higher and more competitive service levels. Producers, usually named factory agents, share their manufacturing assets on the basis of their actual and planned demands or available extra capacities. In [12] a supervised agent-based simulation model was presented to assess the effectiveness of crowdsourced manufacturing.

The current paper, being an inherent continuation of research results published in [12], presents an enhanced distributed collaboration framework developed with the aim of facilitating the cooperation of various production sites. The work encompasses a bi-level simulation model with smart cooperation schemes aiming at managing the resource sharing in a larger, federated network of manufacturers who can dynamically re-configure their mid-term production plans. In our system production planning concerns decisions about mid-term future courses of action that are mostly based on expectations (e.g., demand forecast, resource availability, and material supply). According to such plans and forecasts, production sites formulate their intentions to request or offer capacities, which are communicated to the federation with the main objective to achieve a higher service level and higher utilization ratio of local assets. 
The bi-level simulation includes the factory agent level simulation applied locally at each producer in order to forecast the mid-term capacities of the manufacturing site in question. In our framework the higher level agent-based simulation integrates the overall federation mechanism and supports the analysis of different cooperation scenarios. The remaining part of the paper details this higher level cooperation scheme and presents the first results of the evaluation carried out on randomly generated data set based on an industrial case study.

\section{Formalized model}

Our approach follows the decentralized control paradigm of Industry 4.0: agents are heterogeneous decision makers, which act autonomously and have different KPIs to optimize like financial (e.g., total cost), manufacturing (e.g., Overall Equipment Effectiveness (OEE)) and supply chain (e.g., service level, fill rate). Besides, agents may also have preferences about which partners to choose for collaboration. The Collaboration Platform (CP) serves as a tool for facilitating cooperation among the agents by functioning as a market for resource capacities, see Fig. 1. Agents can offer their free capacities or request capacities when they have surplus or shortages; the platform helps to find suitable offers for each request. Due to the potential complexity of the agents' preferences, the goal of the request-offer matching is not to present one optimal solution for the requesting agent, but to offer some feasible alternatives-similarly to flight search applications that suggest alternative flights by varying search conditions. Accordingly, the final decisions are always on the agents' side.

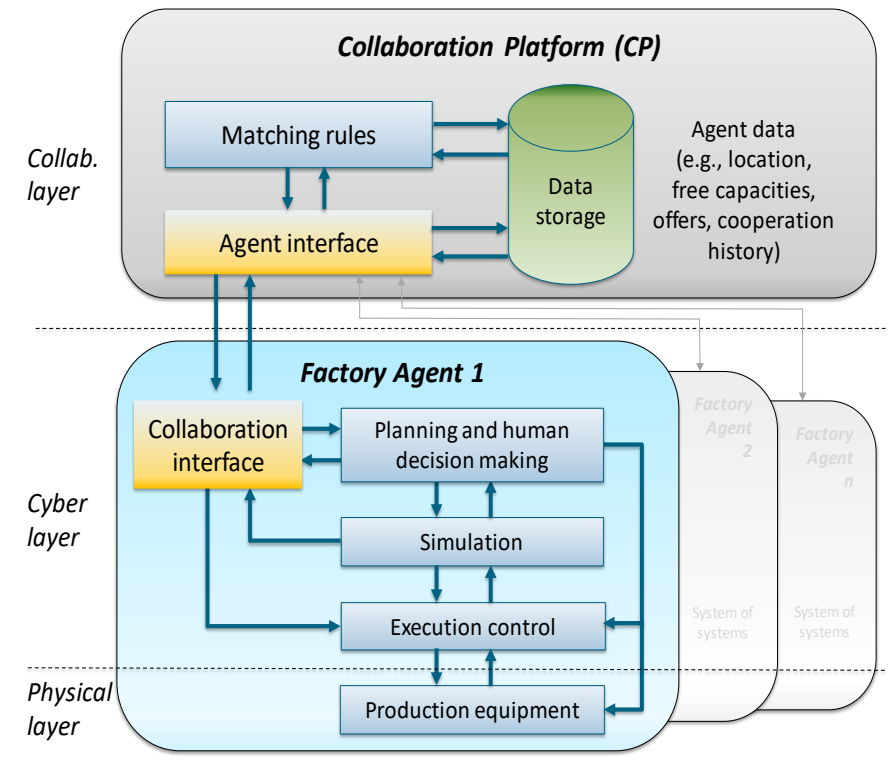

Fig. 1. Layers and components of the model

Fig. 2 presents an overview on the collaboration workflow. Several matching modes are conceivable in this setting, e.g., (i) finding a single offer for the request, (ii) finding a combination of offers that suits the request, (iii) finding requests that not exactly match, but with some tolerance, (iv) asking the other agents whether they can reschedule their production in order to provide capacity offers that match the request, and (v) waiting for future offers. In cases from (i) to (iii), the requesting agent may expect prompt reply from the $\mathrm{CP}$, while in the last two cases (iv) and (v), the reply can be delayed or absent. In the following, we focus on generating the prompt reply, and describe a basic model that formalizes this matching mechanism. It is assumed that the platform stores the incoming offers and whenever a request arrives, it tries to find some alternative proposals from which the requesting agent can choose considering its specific optimization requirements and strategies.
A capacity request can be characterized with the following parameters: $R$ is the resource (or capability), $Q$ is the required quantity, $L$ is the release date of the activity and $U$ is the due date. Capacity offer $i$ is given in a way where: $R_{i}$ is the resource, $L_{i}$ is the earliest start of the activity and $U_{i}$ is the latest finish, $m_{i}$ is the minimum accepted time, $S_{i}$ is the resource speed and $P_{i}$ is the resource utilization price. The role of the parameter $m_{i}$ is to specify a lower limit on the accepted request, e.g., lending capacity for less than a shift is not allowed due to technological or economical reasons. For any request or offer $i$, one can calculate $T T_{i}$, the transportation time, and $T P_{i}$, the price of the (one-way) transportation between the offering and requesting agent.

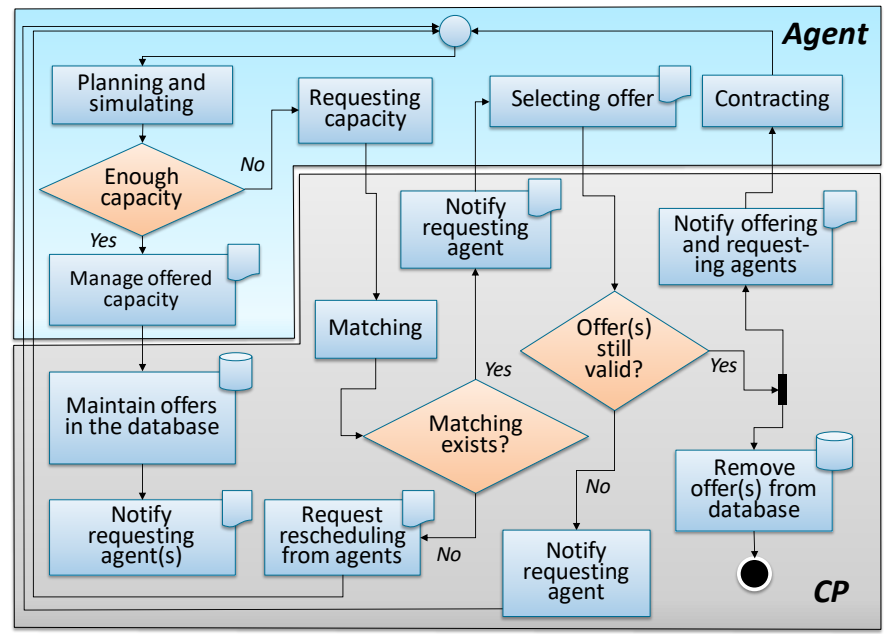

Fig. 2 The flowchart of collaboration

There are three sets of decision variables: $A_{i} \in\{0,1\}$ indicates whether offer $i$ is selected or not, while $s_{i}$ and $e_{i}$ gives the start and end of the production on resource $R_{i}$, respectively. In order to simplify the formalization, two derived variables are also introduced: $T_{i}=e_{i}-s_{i}$ is the production time and $Q_{i}=T_{i} S_{i}$ is the produced quantity on resource $R_{i}$. In order to allow non-exact matches, three additional parameters are used for relaxing the constraints: $\varepsilon_{1}, \varepsilon_{2}, \varepsilon_{3}>0$. The equations also contain an appropriate "big number" $(M)$, a technique frequently utilized in mathematical programming for solving linear problems using the simplex algorithm. The optimization problem can be formalized as follows:

s.t.

$$
\min \sum\left(T_{i} P_{i}+2 A_{i} T P_{i}\right)
$$

$$
\begin{gathered}
\left(R-R_{i}\right) A_{i}=0 \\
L_{i} \leq s_{i} \leq e_{i} \leq U_{i} \\
Q-\varepsilon_{1} \leq \sum Q_{i} \\
\left(L-\varepsilon_{2}+T T_{i}\right) A_{i} \leq s_{i} \\
e_{i}+T T_{i} \leq U+\varepsilon_{3}+\left(1-A_{i}\right) M \\
M A_{i} \geq T_{i} \geq m_{i} A_{i}
\end{gathered}
$$

Eq. (1) expresses that the total production and transportation cost should be minimized. When an offer $i$ is not selected, $A_{i}=0$, thus the transportation cost is not included in the objective function. Furthermore, in this case the production time is zero due to Eq. (7), therefore, it also means zero production cost. Eq. (2) specifies that only the appropriate resource can be matched. Eq. (3) guarantees non-negative production time and that the production fits into the offered interval. Eq. (4) means that the total produced quantity covers the requested quantity minus the allowed shortage. Eq. (5) formulates that there is enough time for the transportation between the release and the start time (with $\varepsilon_{2}$ tolerance). Similarly, Eq. (6) expresses that after the production 
there is enough time for delivering until the deadline with maximum $\varepsilon_{3}$ tardiness. Finally, Eq. (7) ensures that the production time is either zero or exceeds the minimal offered amount (e.g., a shift or a day).

Illustration on Fig. 3 depicts an example where a request is divided between two offers so that the production and the transportation fit into the requested time interval constraints. Since agents are heterogeneous, our model does not include their formal optimization problem but it is open to any type of agents that implements the application and communication interface with the CP (out of scope in this paper).

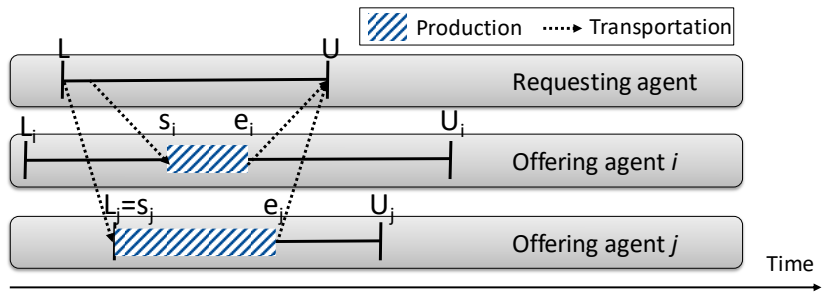

Fig. 3. A possible match between a request and two offers.

\section{Case-study}

\subsection{Cooperation basis in the federation}

Collaboration and negotiation interactions between the factories and the CP have been organized into a use-case scenario, which can be summarized as follows:

- A number of factories federate (by their agents) to the CP by registering and, after running their own simulations and checking for capacity availability, decide whether to offer the resulting capacity on the $\mathrm{CP}$;

- Other factories, on the contrary, may request for the same capacities. Capacity matching cases generate new transactions at the $\mathrm{CP}$, reported to the requesting factories as a list of possible solutions;

- The final contract between two factories (the one offering and the one requesting) is established only after the $\mathrm{CP}$ receives an explicit request (from the requesting factory) to finalize the agreement (with the offering one) for the specific capacity;

- Acknowledging the offering factory implies that the capacity is contracted and must be provided to the requesting factory;

- Capacity may be cancelled by the offering factory if, and only if, they it has not been contracted, yet.

Concerning the formation and operation of the global federation mechanism six main service categories were conceptualized. For each of them we highlight the main execution condition(s) which lead to a successful/unsuccessful conclusion (from the point of view of the factory invoking the request):

1. Factory registration (is prior to any other service invocation by a factory to the CP): (success) new factory with unique agent identifier (URL) asks for registration to the CP; (failure) a factory with the same identifier is found already federated;

2. Factory de-registration: (success) the factory with provided universally unique identifier (UUID) is still a member of the CP; (failure) no factory with the provided UUID is found at the CP;

3. Capacity offer: (success) the factory is currently a member of the CP and the capacity offer is valid (UUIDs for capacity and resources offered are unique). If the offer contains items previously sent to the $\mathrm{CP}$, only new entries are accepted at the federation; (failure) no factory with the provided UUID is found at the $\mathrm{CP}$, or the offer contains only items already registered or with content equivalent to existing ones on the $\mathrm{CP}$;
4. Capacity offer revocation: (success) the factory with provided UUID is a member of the CP and the capacity offer items UUIDs are valid. If offer contained items already sent to the $\mathrm{CP}$, only new entries are added to the federation; (failure) no factory with the provided UUID is found at the $\mathrm{CP}$, or no item in the capacity offer matches with existing ones from the $\mathrm{CP}$, or capacity cannot be cancelled because it has been already contracted;

5. Capacity request: (success) the factory with provided UUID is member of the $\mathrm{CP}$, a capacity request matching is found on the CP for specific (or all) items; (failure) no factory with the provided UUID is found at the $\mathrm{CP}$, or some capacity items have content already registered by a previous capacity request (different UUID but same items), or request contains only already existing items, or no capacity matching item is found at the $\mathrm{CP}$;

6. Contract establishment: (success) the factory is a member of the $\mathrm{CP}$ and negotiable transactions still refer to available resources (no capacity deletion or quicker reply by other factories for the same resource) and offering factories correctly reply to the $\mathrm{CP}$ which has forwarded the request to allocate the capacity for the requesting factory. In this case the contract is considered correctly established; (failure) no factory with provided UUID is found at the CP, or some capacity is no longer available (because deleted or contracted more quickly by other factories), or offering factories do not answer to $\mathrm{CP}$ request to establish the contract, or such transactions are expired or finally, the factory has already an on-going contract established for the specific transaction indicated.

\subsection{Local simulation within a factory agent}

The cooperation framework described above has been implemented and tested with 3 factory agents from a real industrial setting in a distributed cloud environment. Both $\mathrm{CP}$ and factory agents were conceived as a Software as a Service (SaaS) solution and separately deployed onto dedicated Virtual Machines (VMs). In the realized system each agent includes a discrete-event simulation model of the local factory and an interface enabling standardized interactions with the $\mathrm{CP}$ based on the protocol described above. Moreover, factory agents were empowered with a graphical user interface to support the local planning and resource sharing decisions. Additionally, a web-based user interface of the CP allows examining and monitoring the global measures of the cooperation within the federation.

Decisions about requesting and offering capacities were taken locally by using the factory level simulations. An agent has the possibility to execute a "what-if" analysis by using local simulation models. This process can be fully automatized but in a real production environment is usually a supervised planning and forecasting activity carried out by human planners. With the support of the simulation one can analyze and forecast the utilization of each production asset and, in the case of bottlenecks, capacities can be requested. Also when low utilizations are predicted on mid-term horizons capacities can be offered into the federation. Besides the structural and functional details which are described in [12] it is important to mention that the local simulations, together with their main parameters can be constantly mapped and updated with the data acquired from the real world. This is achieved with the Core Manufacturing Simulation Data (CMSD) standard available from Simulation Interoperability Standards Organization (SISO) [13].

\subsection{Experimental results}

In the cloud-based environment mentioned above the real industrial scenario was implemented with the smaller number of 
agents. To test and validate the proposed solution, new collaboration scenarios were developed, which in turn required the creation of several larger federations (composed of 10 to 100 agents). The influence of this cardinality on the cooperation performance and the fluctuations in the utilization of resources and in the service levels were specifically analyzed. In this investigation, a high level multi-agent-based simulation scenario was built with AnyLogic [14]. The system implements a basic version of the matching algorithm presented in Section 2 and a simplified control logic for the agents.

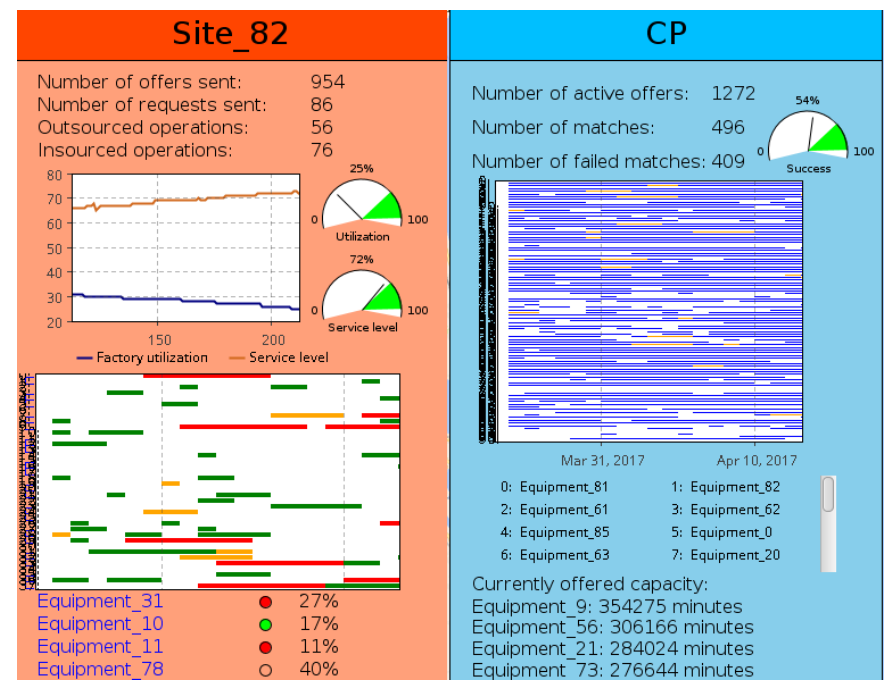

Fig. 4. Dashboard of the high level simulation

The user interface provides a detailed overview of any agent's status, including the utilization of each production equipment, the customer orders delivered in time (service level), as well as information about the collaboration activities, see Fig. 4. The system also shows the status of the CP with the present state of the offers and the past success rate of the request-offer matching.

A federation with 100 agents was generated according to the typical characteristics of the given industry, and the collaboration model was evaluated. Fig. 5 presents the rate of the successful matchings in the function of the participating agents. In the experiments, the more agents offered the capacities, the more probable was to find appropriate offers for a request. Furthermore, the average utilization of the factories has improved with approximately $14 \%$, and the service level with $3 \%$, with no apparent relationship with the number of the agents. This implies higher profitability and better customer satisfaction which are not explicitly calculated for the factory nodes of the federation. Additionally, we suspect that these results might highly depend on the specific characteristics of the production processes, thus they might be different for other industrial sectors. We leave the investigation of the collaboration in other industries for the future work.

\section{Conclusions}

In this paper a federation-based production network model was introduced in which local factory agents cooperate and share their manufacturing assets to achieve a higher level performance in both utilizing resources and increasing service levels.

On the base of a real industrial data-set a cloud-based distributed framework was developed for a smaller number of factories. Applying the same concept, this was scaled up with an order of magnitude and modelled globally as an agent-based simulation in AnyLogic to test how the expansion of the federation influences the cooperation, the variation of service levels and the local utilization of resources.

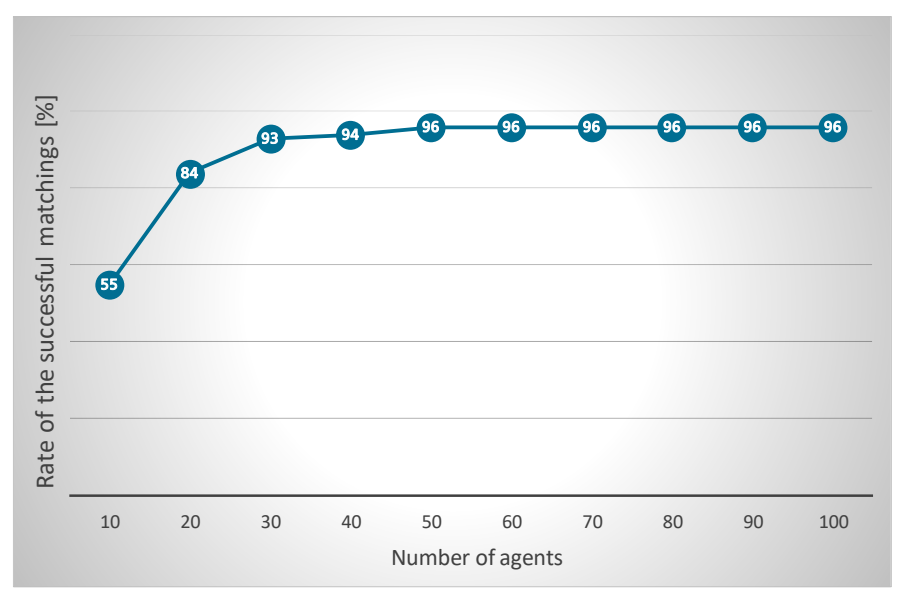

Fig. 5. Rate of the successful matchings

In future works the temporal horizon of the matching algorithm will be extended enabling non-prompt asynchronous feedbacks from the $\mathrm{CP}$ by allowing matchings to happen after additional interactions (see matching options (iv.) and (v.) in Section 2). Additionally, testing new business models (e.g. pay-per-use) by including additional parameters in the cooperation mechanism is also on our research agenda.

\section{Acknowledgement}

This research has been supported by the GINOP-2.3.2-15-201600002 grant on an "Industry 4.0 research and innovation center of excellence" and by the Hungarian Grants OTKA Ref. No. 113038.

\section{References}

[1] Monostori L, Váncza J, Kumara SR (2006) Agent-based systems for manufacturing. CIRP Annals-Manufacturing Technology 55(2):697-720.

[2] Váncza J, Monostori L, Lutters E, Kumara SR, Tseng M, Valckenaers P, Van Brussel H (2011) Cooperative, responsive manufacturing enterprises. CIRP Annals-Manufacturing Technology 60(2):797-820.

[3] Monostori L, Kadar B, Bauernhansl T, Kondoh S, Kumara S, Reinhart G, Sauer O, Schuh G, Sihn W, Ueda K (2016) Cyber-physical systems in manufacturing. CIRP Annals-Manufacturing Technology 65(2):621-641.

44] Camarinha-Matos LM, Afsarmanesh H, Galeano N, Molina A (2009) Collaborative networked organizations:Concepts and practice in manufacturing enterprises. Computers \& Industrial Engineering 57(1):46-60.

[5] Xu X (2012) From cloud computing to cloud manufacturing. Robotics and Computer-Integrated Manufacturing 28(1):75-86

[6] Panetto H, Molina A (2008) Enterprise integration and interoperability in manufacturing systems: Trends and issues. Computers in Industry 59(7):641 646.

[7] Schuh G, Potente T, Wesch-Potente C, Weber AR, Prote J-P (2014) Collaboration mechanisms to increase productivity in the context of Industrie 4.0. Procedia CIRP 19:51-56.

[8] Duffie NA, Roy D, Shi L (2008) Dynamic modeling of production networks of autonomous work systems with local capacity control. CIRP AnnalsManufacturing Technology 57(1):463-466

[9] Freitag M, Becker T, Duffie NA (2015) Dynamics of resource sharing in production networks. CIRP Annals-Manuf. Technology 64(1):435-438

[10] Li B-H, Zhang L, Wang S-L, Tao F, Cao JW, Jiang X, Song X, Chai X (2010) Cloud manufacturing: a new service-oriented networked manufacturing model. Computer integrated manufacturing systems 16(1):1-7.

[11] Mourad M, Nassehi A, Schaefer D (2016) Interoperability as a key enabler for manufacturing in the cloud. Procedia CIRP 52:30-34.

[12] Kaihara T, Katsumura Y, Suginishi Y, Kadar B (2017) Simulation model study for manufacturing effectiveness evaluation in crowdsourced manufacturing CIRP Annals-Manufacturing Technology 66(1):445-448.

[13] (SISO) SISO (visited: 16th January 2018) Standard for: Core Manufacturing Simulation Data. [Online]. https://www.sisostds.org/ ProductsPublications/Standards/SISOStandards.aspx

[14] Borshchev A (2013) The big book of simulation modeling: multimethod modeling with AnyLogic 6, AnyLogic North America. 